\title{
Atelectasia pulmonar em cães durante anestesia geral
}

\author{
Pulmonary atelectasis in dogs during general anesthesia
}

\author{
Patrícia Cristina Ferro Lopes ${ }^{\mathrm{I}}$ Newton Nunes ${ }^{\mathrm{II}}$
}

\section{RESUMO}

A pressão intrapleural normalmente é menor que a pressão intrapulmonar. Consequentemente, os pulmões tendem ao colapso e se retraem, afastando-se da parede torácica. No início do século XX, Pasteur descreveu a atelectasia pulmonar, que ocorre com frequência durante a indução anestésica, persiste no período pós-operatório e pode contribuir de maneira significativa para a morbidade e o aumento nos gastos com medicamentos. Em medicina veterinária, no entanto, a atelectasia não é frequentemente diagnosticada, apesar de que isso não implica afirmar que tal afecção não ocorra, visto que existem relatos do desenvolvimento desse quadro em cães e em outras espécies. No contexto da anestesia geral, essa complicação pulmonar pode ser encontrada em animais que respiram 80 a 100\% de oxigênio. A partir dessas informações, torna-se necessário que o profissional da anestesiologia veterinária obtenha conhecimentos complementares sobre o tema. Com este trabalho, objetivouse descrever alguns dos mecanismos da atelectasia e seus pontos relevantes, de modo a familiarizar os profissionais quanto aos pormenores dessa importante, e nem sempre bem compreendida, alteração fisiológica respiratória.

Palavras-chave: colapso pulmonar, fração inspirada de oxigênio, shunt.

\section{ABSTRACT}

Pleural pressure is usually lower than pulmonary pressure. Therefore, the lungs tend to collapse and increase its distance from thoracic walls. At the beginning of $20^{\text {th }}$ century, Pasteur described the pulmonary atelectasis, which develops during induction of anesthesia and persists to the postoperative period. It can contribute significantly to morbidity and to increase the medical expenses. In veterinary medicine, pulmonary atelectasis is not frequently diagnosed, which doesn't rule out the occurrence of this disease, since there are reports of atelectsasis in dogs. This pulmonary complication can be found in animals that breathe $80 \%$ to $100 \%$ oxygen in anesthetic procedures. Based on this information, the veterinary anesthesiologist is required to search for complementary knowledge regarding to pulmonary atelectasis. This study, therefore, aimed to describe some of the mechanisms involved in the development of atelectasis. It aimed also to familiarize the anesthesiologist to this not always well- understood physiological breathing change.

Key words: fractional inspired oxygen, pulmonary collapse, shunt.

\section{INTRODUÇÃO}

O termo atelectasia significa distensão incompleta dos alvéolos e é utilizado para descrever o pulmão que não se expandiu com o ar por ocasião do nascimento (atelectasia congênita) ou que colapsou após ter sido inflado (atelectasia adquirida) (LÓPEZ, 1998).

No início do século XX, PASTEUR (1910) descreveu a atelectasia pulmonar, sendo esta uma enfermidade que ocorre com frequência durante a

\footnotetext{
IPrograma de Pós-graduação em Cirurgia Veterinária, Faculdade de Ciências Agrárias e Veterinárias (FCAV), Universidade Estadual Paulista (UNESP), Jaboticabal, SP, Brasil.

IIDepartamento de Clínica e Cirurgia Veterinária, FCAV, UNESP. Via de Acesso Prof. Paulo Donato Castellane, s/n, Zona Rural, 14884-900, Jaboticabal, SP, Brasil. E-mail: newton@fcav.unesp.br. Autor para correspondência.
} 
indução anestésica (LORING \& BUTLER, 1987), persiste no período pós-operatório (LAWRENCE et al., 1995) e pode contribuir de maneira significativa para a morbidade (ROTHEN et al., 1993) e o aumento do período de recuperação, com consequente elevação nos custos do tratamento do paciente (LAM et al., 1998).

Em seu trabalho clássico, BENDIXEN et al. (1963) propuseram que a atelectasia seria uma das causas de prejuízo da oxigenação durante a anestesia. Esses autores observaram sucessiva diminuição da complacência do sistema respiratório e coincidente redução da oxigenação arterial em humanos e animais anestesiados, fato interpretado como formação de atelectasia, a qual pode ser encontrada em diferentes níveis de acordo com a área pulmonar, existindo correlação entre a presença de shunt e a área de colapso pulmonar (BRISMAR et al., 1985; GUNNARSSON et al., 1991).

Por volta de 1980, com a utilização da tomografia computadorizada, áreas de atelectasia foram diagnosticadas em pacientes humanos anestesiados (BRISMAR et al., 1985), sendo observado o rápido desenvolvimento de densidades diferentes nos pulmões durante o procedimento anestésico. Estudos morfológicos de densidades pulmonares iguais, observadas em várias ovelhas, puderam confirmar o diagnóstico de atelectasia (HEDENSTIERNA et al., 1989).

AKÇA et al. (1999), estudando seres humanos anestesiados recebendo 30 ou $80 \%$ de oxigênio $\left(\mathrm{O}_{2}\right)$, utilizaram a radiografia torácica como um dos meios de diagnóstico de atelectasia, enquanto ALDRICH et al. (2002), por meio da broncoscopia, detectaram severo colapso das vias aéreas durante procedimento anestésico em um cão. Áreas de atelectasias ainda podem ser diagnosticadas por ressonância magnética, ultrasonografia, perfil de citocinas e microscopia intravital. Suspeitas características do desenvolvimento de colapso pulmonar, quando ocorrem alterações na fisiologia pulmonar como diminuição da complacência ou prejuízo na oxigenação, são descritas por DUGGAN \& KAVANAGH(2005).

Durante a anestesia geral, em animais respirando frações inspiradas de oxigênio $\left(\mathrm{FiO}_{2}\right)$ de 1,0 ou 0,8, pode-se observar a ocorrência de atelectasia (HEDENSTIERNA et al., 1989; JEFFERIES, 1994), que também foi sugerida em cães submetidos à infusão contínua de propofol e mantidos em ventilação espontânea com as mesmas $\mathrm{FiO}_{2}$ supracitadas (LOPES et al., 2007). Em animais, o colapso pulmonar prolongado pode resultar em abscedação e fibrose, sendo necessária a intervenção cirúrgica se os sinais persistirem após a correção do problema subjacente (HAWKINS, 1997).

A partir dessas informações, torna-se necessário, para o profissional da anestesiologia veterinária, obter mais conhecimentos sobre esse tema, apesar de ainda não ser estudado amplamente. Portanto, este trabalho descreverá a seguir alguns dos mecanismos de atelectasia e seus pontos relevantes, para que se possa avançar nessa área da medicina veterinária.

Anestesia geral e atelectasia

A atelectasia induzida pela anestesia é tradicionalmente conhecida como colapso pulmonar (DUGGAN \& KAVANAGH, 2005) e pode ser desenvolvida em anestesias gerais inalatórias ou intravenosas, quando o paciente está respirando espontaneamente ou está sendo ventilado mecanicamente (STRANDBERG et al., 1986). O único anestésico testado que não produz atelectasia é a cetamina (TOKICS et al., 1987). Entretanto, quando esse fármaco é administrado em associação a um bloqueador neuromuscular e o paciente for submetido à ventilação mecânica, o colapso pode surgir. Por outro lado, a anestesia epidural causa nenhuma ou pouca área de atelectasia e não provoca alterações no shunt e na taxa de ventilação/perfusão (REBER et al., 1998). Além da anestesia, HAWKINS (1997) afirmou que o decúbito prolongado ou a diminuição da atividade ciliar pode potencializar a incidência de colapso alveolar.

BENDIXEN et al. (1963) postularam que a ventilação espontânea, sem respirações profundas periódicas, pode conduzir para formação progressiva de áreas de atelectasia, com aumento do shunt e diminuição da complacência pulmonar, e que essas alterações são reversíveis quando se hiperinfla os pulmões. Os autores demonstraram que a anestesia geral, sem suplementação de $\mathrm{O}_{2}$, reduz a pressão parcial de oxigênio no sangue arterial $\left(\mathrm{PaO}_{2}\right)$ em $22 \%$ e a complacência em $15 \%$, e que três hiperinflações sucessivas dos pulmões restabeleceram esses parâmetros, sugerindo, dessa forma, que respirações periódicas profundas previnem a atelectasia progressiva e o shunt.

Em cães submetidos à anestesia intravenosa com propofol e mantidos em respiração espontânea, atribuiu-se a formação de áreas de atelectasia à diminuição da contratilidade do diafragma, causada pelo fármaco administrado, associado ao emprego de altas $\mathrm{FiO}_{2}$ (1 e 0,8) (LOPES et al., 2007). Os mesmos autores observaram hipercapnia, que é comumente relacionada aos colapsos pulmonares (JEFFERIES, 1994), pois a 
pressão parcial de dióxido de carbono no sangue arterial $\left(\mathrm{PaCO}_{2}\right)$ é um valor de referência usado para avaliar a ventilação alveolar e a detecção precoce da formação de atelectasia (MILLER, 2005).

No período pós-operatório, ALDRICH et al. (2002) observaram o desenvolvimento de hipoxemia e severa atelectasia em um cão. Segundo BRAINARD et al. (2006), caninos com risco anestésico ASA=III que necessitem de anestesia de urgência ou de longa duração têm alta probabilidade de desenvolver complicações pulmonares pós-operatórias.

Mecanismos de formação de atelectasia durante a anestesia

A atelectasia pulmonar pode ser causada por vários fatores, os quais devem ser classificados em três mecanismos básicos: a atelectasia compressiva que ocorre quando a pressão trasmural, responsável pela dilatação dos alvéolos, está reduzida; a por absorção que surge quando a quantidade de gás que entra nos alvéolos é menor que aquela removida pela transferência para o sangue; e, por último, a atelectasia por perda de surfactante que aparece quando a tensão superficial dos alvéolos aumenta devido à redução da ação do surfactante (HEDENSTIERNA, 2003; MAGNUSSON \& SPAHN, 2003; DUGGAN \& KAVANAGH, 2005).

\section{Atelectasia por compressão}

O diafragma separa dois compartimentos (cavidade torácica e abdominal), com diferentes pressões, bem como gradientes de pressões verticais. Assim, a pressão final expiratória é normalmente mais baixa que a pressão abdominal. Se essa musculatura não estiver rígida o suficiente entre as duas cavidades, a pressão abdominal será transmitida para o interior da cavidade torácica, aumentando, em particular, a pressão pleural nas regiões pulmonares e resultando em atelectasia (HEDENSTIERNA, 2003). Evidência indireta da veracidade da assertiva é o fato de a anestesia com cetamina não causar atelectasia (TOKICS et al., 1987), já que esse fármaco apresenta a característica de manter a função muscular respiratória. Assim, a importância do diafragma é clara na redução da taxa de colapso alveolar (HEDENSTIERNA et al., 1994). Todavia, em cães, outros anestésicos, como o propofol, causam diminuição na contratilidade do diafragma (FUJII et al., 2001).

CARARETO (2007), em seu estudo com cães anestesiados com propofol e sufentanil, mantidos em ventilação controlada, registrou valores elevados de shunt, atribuindo tal fato ao posicionamento dos animais na mesa cirúrgica, sendo adotada a posição de
Trendenlemburg a 30 graus. De acordo com a autora, essa posição proporcionou o deslocamento da cúpula diafragmática, com redução do diâmetro torácico e decréscimo da capacidade residual funcional (CRF), sugerindo, portanto, a formação de áreas de colapso pulmonar. Segundo HENDENSTIERNA (2003), o aumento na $\mathrm{FiO}_{2}$ pode promover a formação de atelectasia se houver concomitante redução da CRF.

Na espécie canina, o colapso pulmonar varia diretamente com a diminuição dos valores da pressão intrapleural, e valores entre -3 e $-12 \mathrm{~cm} \mathrm{H}_{2} \mathrm{O}$ proporcionam redução das áreas de atelectasia, enquanto que valores entre $-7,5 \mathrm{e}-24 \mathrm{~cm} \mathrm{H}_{2} \mathrm{O}$ produzem aumento no número de regiões colapsadas (FINLEY et al., 1963).

FROESE \& BRYAN (1974) demonstraram que o movimento diafragmático, em pacientes com respiração espontânea normal, é alterado quando os agentes bloqueadores neuromusculares são administrados. Do mesmo modo, KRAYER et al. (1989), usando tomografia computadorizada, encontraram alteração no movimento diafragmático durante a anestesia geral e ventilação mecânica. Assim, a atelectasia por compressão ocorre durante a anestesia geral e é causada pela geometria, posição e movimentação do diafragma (MAGNUSSON \& SPAHN, 2003).

\section{Atelectasia por absorção}

Estudos clínicos indicam que a atelectasia por absorção tem grande influência na gênese dos colapsos alveolares durante a anestesia (ROTHEN et al., 1995a), e esta pode ocorrer por dois mecanismos. O primeiro acontece após a oclusão completa das vias aéreas, criando-se uma bolsa de ar retido na porção distal da unidade pulmonar obstruída. A pressão nessa bolsa, inicialmente, está próxima da pressão atmosférica. A mistura de sangue venoso que perfunde essa região de gás retido possui pressão de gás parcial inferior à pressão atmosférica, ou seja, apresenta uma pressão subatmosférica. Dessa forma, o gás transfere-se da bolsa para o sangue continuamente, até a bolsa se colapsar (LORING \& BUTLER, 1987).

O segundo mecanismo ocorre na ausência de obstrução das vias aéreas. Nesse contexto, áreas pulmonares têm a taxa de ventilação/perfusão $\left(\mathrm{V}_{\mathrm{A}} / \mathrm{Q}\right)$ menor que o valor crítico. Esse valor é conhecido como sendo o ponto no qual a quantidade de gás que entra nos alvéolos é igual àquela que é transferida para o sangue (DANTZKER et al., 1974), ou seja, apresentam $\mathrm{V}_{\mathrm{A}} / \mathrm{Q}$ e pressão parcial alveolar de oxigênio $\left(\mathrm{P}_{\mathrm{A}} \mathrm{O}_{2}\right)$ baixas. Quando a $\mathrm{FiO}_{2}$ é aumentada, a $\mathrm{P}_{\mathrm{A}} \mathrm{O}_{2}$ aumenta, causando grande aumento na taxa de transferência do 
oxigênio alveolar para o sangue capilar. $\mathrm{O}$ fluxo de $\mathrm{O}_{2}$ pode aumentar tanto que a transferência do gás para o sangue excede o fluxo de gás inspirado, e a unidade pulmonar torna-se progressivamente menor. É mais comum ocorrer o colapso quando a $\mathrm{FiO}_{2}$ e o tempo de exposição são altos ou em áreas que a $\mathrm{V}_{\mathrm{A}} / \mathrm{Q}$ é baixa (REHDER et al., 1979). Reciprocamente, a redução na área de atelectasia é vista quando baixa concentração de oxigênio é utilizada na indução (ROTHEN et al., 1996), durante a manutenção da anestesia (JEFFERIES, 1994) ou apenas antes da extubação (BENOÎT et al., 2002).

A $\mathrm{FiO}_{2}$ e a solubilidade do gás inerte na mistura inspirada são fatores determinantes para a taxa de absorção da mistura gasosa. Se as misturas de gases inspiradas contêm um gás relativamente insolúvel, como o nitrogênio, a taxa de transferência do gás é relativamente lenta, e quando há aumento da $\mathrm{FiO}_{2}$, esta se torna mais rápida. Quando o gás inspirado contém oxigênio e gás inerte relativamente solúvel, como óxido nitroso, a transferência do gás é mais rápida (JOYCE et al., 1993). JOYCE et al. (1996) observaram, em seu estudo com cães, que, quando se utiliza uma mistura de oxigênio $\left(\mathrm{FiO}_{2}>0,3\right)$ e óxido nitroso, a taxa de transferência de gás de um pulmão não ventilado é mais rápida que com a utilização de oxigênio a 100\%. Isso implica que essa mistura gasosa acelera a atelectasia por absorção. Portanto, a escolha da mistura gasosa a ser utilizada na anestesia pode auxiliar na prevenção de áreas de colapso alveolar.

Atelectasia por perda do surfactante

O surfactante é sintetizado no reticuloendoplasmático e armazenado nos corpos lamelares dos pneumócitos do tipo II; quando os alvéolos são submetidos a uma série de estímulos, este é secretado. Nessa região, o surfactante desempenha sua principal função: diminuir a tensão superficial para manter a estabilidade alveolar (BEPPU, 1995).

A ação do surfactante pode ser prejudicada pela anestesia. Tal afirmativa foi comprovada por WOLLMER et al. (1990), os quais, trabalhando com clerance pulmonar do ácido dietileno-triamino-penta acético marcado com tecnésio 99 ${ }^{\mathrm{m}}$ (DTPA-99mTc), demonstraram que a anestesia com halotano associada a altas $\mathrm{FiO}_{2}$ aumentam a permeabilidade da barreira alvéolo-capilar do pulmão de coelhos. Esses autores propuseram que o aumento da taxa de clerance do DTPA-99mTc, durante a anestesia com halotano, foi provavelmente causado pelos efeitos sobre o surfactante pulmonar, ou o epitélio alveolar ou ambos. Além disso, é sabido que o conteúdo do surfactante alveolar é modificado por fatores mecânicos (DUGGAN \& KAVANAGH, 2005). A ausência de respirações profundas intermitentes, como ocorre geralmente na ventilação mecânica, pode resultar na redução de conteúdos ativos de surfactante alveolar (OTIS et al., 1993), que tem como consequência uma redução da estabilidade alveolar, podendo contribuir para o acúmulo de líquido no lúmen das vias aéreas, e assim causar obstrução das mesmas (OYARZUN et al., 1991).

ENNEMA et al. (1984), em estudo com coelhos, observaram que, durante a ventilação, o surfactante alveolar é inativado, mas é reversível com um único e largo ato de inflar o pulmão, denominada manobra de capacidade-vital (MCV) (BENDIXEN et al., 1963; ROTHEN et al., 1993). A MCV pode promover a produção ou liberação do surfactante e distribuição deste sobre a superfície alveolar, podendo, dessa forma, proteger contra possíveis novos colapsos. No entanto, em seu estudo com pulmões de cães, FARIDY (1976) sugeriu que o oxigênio a $100 \%$ parece ter um efeito inibitório nesse processo de produção ou liberação de surfactante.

Os três mecanismos citados podem contribuir para formação de atelectasia durante a anestesia geral. No entanto, alterações primárias na tensão de superfície são menos importante devido à reserva e ao período de 14 horas, necessário à renovação do surfactante. Dessa forma, absorção e compressão são considerados os dois mecanismos que mais influenciam a formação de atelectasia (MAGNUSSON \& SPAHN, 2003).

Fatores relacionados a áreas de atelectasia

Altas concentrações de $\mathrm{O}_{2}$ têm sido associadas com a formação de áreas de atelectasia (MAGNUSSON \& SPAHN, 2003). O fato se reveste de importância, pois altas $\mathrm{FiO}_{2}$ são comumente empregadas pelos anestesiologistas. Para evitar esse problema, baixas concentrações desse gás têm sido utilizadas durante a indução da anestesia geral. Com $100 \%$ de oxigênio, o shunt aumenta de 0,3 para $6,5 \%$, com formação de atelectasia correspondente a uma área de $8 \mathrm{~cm}^{2}$ em seres humanos (AKÇA et al., 1999). Já com oxigênio a $30 \%$, o shunt aumenta somente 2,1\%, com uma área mínima de atelectasia de $0,2 \mathrm{~cm}^{2}$ (ROTHEN et al., 1996).

Na ausência da pré-oxigenação, o colapso pulmonar não tem sido observado logo após a indução da anestesia; embora, quando $\mathrm{FiO}_{2}$ é aumentada para 1,0 depois da intubação, a atelectasia torna-se um achado frequente (ROTHEN et al., 1995b). O fato de o $\mathrm{O}_{2}$ induzir colapsos alveolares não está restrito apenas à indução, pois aumentos de $\mathrm{FiO}_{2}$ ao final da cirurgia e antes da extubação podem causar atelectasia adicional (BENOÎT et al., 2002). 
LOPES (2005) estudou o efeito de diferentes $\mathrm{FiO}_{2}$ em cães submetidos à infusão contínua de propofol e mantidos em ventilação espontânea. Nessa ocasião, concluiu-se que o fornecimento de oxigênio a 100 e $80 \%$ deve ser evitado, pois determina alterações significativas no sistema respiratório e, complementarmente, sugeriu-se a formação de áreas de atelectasia pulmonar com o emprego dessas concentrações de $\mathrm{O}_{2}$. No mesmo estudo, a $\mathrm{FiO}_{2}$ de 0,21 proporcionou alterações significativas indesejáveis na oxigenação de animais da espécie canina sendo, portanto, indicado o emprego de $\mathrm{FiO}_{2}=0,6 \mathrm{em}$ procedimentos com infusão contínua desse fármaco. Segundo NUNN (1979), quando administradas $\mathrm{FiO}_{2}$ maiores que 0,6 , o nitrogênio, gás responsável por manter os alvéolos abertos em regiões de baixa taxa de $\mathrm{V}_{\mathrm{A}} / \mathrm{Q}$, é removido, causando colapso.

$\mathrm{O}$ aumento de $\mathrm{FiO}_{2}$ é relacionado com o aumento da ocorrência de shunt pulmonar (ROSA et al., 2006) e altos níveis de atelectasia (MAGNUSSON \& SPAHN, 2003). ROSA et al. (2006), empregando anestesia inalatória com sevofluorano e ventilação controlada, registraram maior grau de shunt pulmonar em cães submetidos ao sistema de anestesia sem reinalação com $\mathrm{FiO}_{2}=0,9$ que nos animais mantidos no sistema de anestesia com reinalação e $\mathrm{FiO}_{2}=0,4$.

Ressalta-se que, juntamente com $\mathrm{FiO}_{2}$, a composição do gás a ser administrado tem papel importante na contribuição da formação de áreas de atelectasia e na alteração da relação ventilação/ perfusão (ROTHEN et al., 1995b). Em cães, quando se utiliza uma mistura de oxigênio e óxido nitroso com $\mathrm{FiO}_{2}>0,3$, a taxa de transferência de gás de um pulmão não ventilado é mais rápida que com a utilização de oxigênio a 100\% (JOYCE et al.,1996).

Recentemente, durante anestesia geral, foi demonstrado que pacientes humanos com obesidade apresentavam áreas mais intensas de atelectasia quando comparados com os demais indivíduos, (EICHENBERGER et al., 2002). A CRF é menor em pacientes obesos, pois o gradiente de oxigenação alveolar-arterial ( $\left.\mathrm{A}-\mathrm{aDO}_{2}\right)$ é maior, e a pressão intraabdominal é mais alta. Os diferentes mecanismos do sistema respiratório e a hipóxia encontrados nesses pacientes podem ser consequência da redução do volume pulmonar causada pela alta pressão intraabdominal (PELOSI et al., 1997). A formação de áreas de colapso alveolar pode ser encontrada em todas as idades (GUNNARSSON et al., 1991).

Segundo ROSA et al. (2006), a umidificação dos gases durante a ventilação controlada em cães com $\mathrm{FiO}_{2}=0,4$ ajuda a diminuir o shunt. Isso porque, durante a anestesia geral, por meio do tubo traqueal, chega até os alvéolos um ar frio e seco, tornando o muco de revestimento menos úmido e mais denso e levando à obstrução das vias aéreas.

Apesar de existirem diferentes fatores que podem proporcionar o aparecimento de áreas de atelectasia, segundo HEDENSTIERNA (2003), há várias intervenções que podem ajudar na prevenção ou na reabertura dos tecidos colapsados, entre elas estão a manutenção ou restauração do tônus muscular respiratório, a MCV, a minimização da reabsorção pulmonar do gás e a pressão positiva no final da expiração (PEEP), que, quando aplicada em pulmões comprometidos, promove significativa redução do shunt e melhora da oxigenação arterial (SENTÜRK, 2006). Contudo, ao empregar crescentes $\operatorname{PEEP}(0,5$, 10cm $\mathrm{H}_{2} \mathrm{O}$ ), CARARETO (2007) não observou melhora no shunt em cães anestesiados com propofol e sufentanil, mantidos em ventilação controlada e posicionados em cefalodeclive.

Como descrito anteriormente, a MCV pode eliminar as áreas de atelectasia na maioria dos pacientes e restabelecer a troca gasosa normal. No entanto, essas áreas podem reaparecer dentro de alguns minutos se for utilizado oxigênio a 100\%, mas isso não ocorre ser for aplicado simultaneamente uma PEEP igual a $10 \mathrm{~cm}$ $\mathrm{H}_{2} \mathrm{O}$. Assim, a MCV seguida por PEEP de $10 \mathrm{~cm} \mathrm{H}_{2} \mathrm{O}$ deve ser utilizada quando pacientes são ventilados com altas frações de oxigênio inspirado e quando a troca gasosa estiver prejudicada (NEUMANN et al., 1999).

\section{CONSIDERAÇÕES FINAIS}

É possível sugerir que áreas de atelectasia durante os procedimentos anestésicos em cães ocorrem, mas não se pode afirmar a frequência e a gravidade para esses pacientes. No entanto, cabe ressaltar que o aumento do shunt, a hipercapnia e a diminuição da complacência pulmonar e da $\mathrm{PaO}_{2}$ podem indicar a presença de colapso pulmonar nos animais durante a anestesia. Nesses casos, o emprego de baixas $\mathrm{FiO}_{2}$, da MCV e da PEEP podem auxiliar o anestesiologista a controlar esse problema. Contudo, são necessários estudos mais detalhados sobre esse assunto, para definir as técnicas que poderão prevenir a formação de áreas de atelectasia em cães.

\section{AGRADECIMENTOS}

Os autores agradecem à Fundação de Amparo à Pesquisa do Estado de São Paulo (FAPESP), pela concessão da bolsa de doutorado para Patrícia Cristina Ferro Lopes. 


\section{REFERÊNCIAS}

AKÇA, O. et al. Comparable postoperative pulmonary atelectasis in patients given $30 \%$ or $80 \%$ oxygen during and 2 hours after colon resection. Anesthesiology, Philadelphia, v.91, n.4, p.991-998, 1999. Disponível em: <http:// journals.lww.com/anesthesiology/Fulltext/1999/10000/ Comparable_Postoperative_Pulmonary_Atelectasis_in.19.aspx>. Acesso em: 8 set. 2009.

ALDRICH, J. et al. Successful ventilatory management of post-anesthetic airway collapse and hypoxemia in a dog. Journal of Veterinary Emergency and Critical Care, Columbus, v.12, n.2, p.105, 2002. Disponível em: <http:// www3.interscience.wiley.com/cgi-bin/fulltext/118963986/ PDFSTART>. Acesso em: 8 set. 2009. doi: 10.1046/j.14356935.2002.00024.x.

BENDIXEN, H.H. et al. Impaired oxygenation in surgical patients during general anaesthesia with controlled ventilation: a concept of atelectasis. New England Journal of Medicine, Boston, v.290, n.269, p.991-996, 1963.

BENOÎT, Z. et al. The effect of increased $\mathrm{FiO}_{2}$ before tracheal extubation on postoperative atelectasis. Anesthesia and Analgesia, Baltimore, v.95, n.6, p.1777-1781, 2002. Disponível em: <http://www.anesthesia-analgesia.org/cgi/ content/full/95/6/1777>. Acesso em: 8 set. 2009.

BEPPU, O.S. Surfactante pulmonar. Composição, função e metabolismo. In: AULER, J.O.C.; AMARAL, R.V.G. Assistência ventilatória mecânica. São Paulo: Atheneu, 1995. p.49-61.

BRAINARD, B.M. et al. Postoperative pulmonary complications in dogs undergoing laparotomy: anesthetic and perioperative factors. Journal of Veterinary emergency and Critical Care, Columbus, v.16, n.3, p.184-191, 2006. Disponível em: <http://www3.interscience.wiley.com/cgi-bin/ fulltext/118556740/ PDFSTART>. Acesso em: 8 set. 2009. doi: 10.1111/j.1476-4431.2006.00177.x.

BRISMAR, B. et al. Pulmonary densities during anaesthesia with muscular relaxation: a proposal of atelectasis. Anesthesiology, Philadelphia, v.62, n.4, p.422-428, 1985. Disponível em: < http://journals.lww.com/anesthesiology/toc/ 1985/04000>. Acesso em: 8 set. 2009.

CARARETO, R. Ventilação controlada a volume ou a pressão em cães anestesiados com infusão contínua de propofol e sufentanil, mantidos em cefalodeclive e submetidos a diferentes pressões positivas expiratórias finais. 2007. 176f. Tese (Doutorado em Cirurgia Veterinária) - Faculdade de Ciências Agrárias e Veterinárias, Universidade Estadual Paulista, Jaboticabal, SP.

DANTZKER, D.R. et al. Proceedings: Instability of poorly ventilated lun units during oxygen breathing. Journal of Physiology, Cambridge, v.242, n.2, p.72, 1974.

DUGGAN, M.; KAVANAGH, B.P. Pulmonary atelectasis: a pathogenic perioperative entity. Anesthesiology, Philadelphia, v.102, n.4, p.838-854, 2005. Disponível em: http:// journals.lww.com/anesthesiology/toc/2005/04000>. Acesso em: 8 set. 2009.
EICHENBERGER, A.S. et al. Morbid obsety and postoperative pulmonary atelectasis: na underestimated problem. Anesthesia and Analgesia, Baltimore, v.95, n.6, p.1788-1792, 2002. Disponível em: <http://www.anesthesia-analgesia.org/cgi/ content/full/95/6/1788>. Acesso em: 8 set. 2009. doi: 10.1213/ 01.ANE.0000035081.21607.4C.

ENNEMA, J.J. et al. Effects of artificial ventilation on surfactant phospholipid metabolism in rabbits. Respiration Physiology, Netherlands, v.58, n.1, p.15-28, 1984. Disponível em: <http:// www.sciencedirect.com/science/journal/00345687>. Acesso em: 8 set. 2009. doi: 10.1016/0034-5687(84)90041-0.

FARIDY, E.E. Effect of distension on release of surfactant in excised dog's lungs. Respiration Physiology, Netherlands, Disponível em: <http://www.sciencedirect.com/science/journal/ 00345687>. Acesso em: 8 set. 2009. doi:10.1016/00345687(76)90021-9.

FINLEY, T.N. et al. Effect of intrapleural pressure on pulmonary shunt through atelectatic dog lung. American Journal of Physiology, Bethesda, v.205, p.1187-1192, 1963. Disponível em: <http://ajplegacy.physiology.org/cgi/reprint/205/ 6/1187>. Acesso em: 8 set. 2009.

FROESE, A.B.; BRYAN, A.C. Effects of anesthesia and paralysis on diaphragmatic mechanics in man. Anesthesiology, Philadelphia, v.41, n.3, p.242-255, 1974. Disponível em: <http:/ /journals.lww.com/anesthesiology/toc/1974/09000>. Acesso em: 8 set. 2009.

FUJII, Y. et al. The dose-range effects of propofol on the contractility of fatigued diaphragm in dogs. Anesthesia and Analgesia, Baltimore, v.93, n.5, p.1194-1198, 2001. Disponível em: <http://www.anesthesia-analgesia.org/cgi/ content/full/93/5/1194>. Acesso em: 8 set. 2009.

GUNNARSSON, L. et al. Influence of age on atelectasis formation and gas exchange impairment during general anaesthesia. British Journal of Anaesthesia, Oxford, v.66, n.4, p.423-432, 1991. Disponível em: <http://bja.oxfordjournals.org/cgi/reprint/66/4/ 423>. Acesso em: 8 set. 2009.

HAWKINS, E.C. Afecções do sistema respiratório. In: ETTINGER, S.; FELDMAM, E.C Tratado de medicina interna veterinária. 4.ed. São Paulo: Manole, 1997. p.10801142 .

HEDENSTIERnA, G. et al. Pulmonary densities during anaesthesia. Na experimental study on lung histology and gas exchange. European Respiratory Journal, Leiden, v.2, n.6, p.528-535, 1989.

HEDENSTIERNA, G. et al. Phrenic nerve stimulation during halothane anaesthesia. Anesthesiology, Philadelphia, v.80, n.4, p.751-760, 1994. Disponível em: <http://journals.lww.com/ anesthesiology/toc/1994/04000>. Acesso em: 8 set. 2009.

HEDENSTIERNA, G. Alveolar collapse and closure of airways: regular effects of anaesthesia. Clinical Physiology and Functional Imaging, Oxford, v.23, n.3, p.123-129, 2003. Disponível em: <http://www3.interscience.wiley.com/ journal/ 118899823>. Acesso em: 8 set. 2009. doi: 10.1046/j.1475097X.2003.00483.x. 
JEFFERIES, A.R. Pathology. In: HALL, L.W.; TAYLOR, P.M. Anaesthesia of the cat. London: Baillière Tindall, 1994. p.63-88.

JOYCE, C.J. et al. Gas uptake from na unventilated area of lung: computer model of absorption atelectasis. Journal of Applied Physiology, Bethesda, v.74, n.3, p.1107-1116, 1993. Disponível em: <http://jap.physiology.org/cgi/content/abstract/ 74/3/1107>. Acesso em: 8 set. 2009.

JOYCE, C.J. et al. Nitrous oxide and the rate of gas uptake from an unventilated lun in dogs. British Journal of Anaesthesia, Oxford, v.76, n.2, p. 292-296, 1996. Disponível em: < http://bja.oxfordjournals.org/cgi/reprint/76/2/292.pdf>. Acesso em: 8 set. 2009.

KRAYER, S. et al. Position and motion of the human diaphragm during anesthesia-paralysis. Anesthesiology, Philadelphia, v.70, n.6, p.891-898, 1989. Disponível em: <http://journals.lww.com/ anesthesiology/toc/1989/06000>. Acesso em: 8 set. 2009.

LAM, W.W.M. et al. Sedation versus general anaesthesia in paediatric patients undergoing chest CT. Acta Radiologica, Stockholm, v.39, n.3, p.298-300, 1998.

LAWRENCE, V.A. et al. Incidence and hospital stay for cardiac and pulmonary complications after abdominal surgery. Journal of General Internal Medicine, New York, v.10, n.12, p.671678, 1995. Disponível em: <http://www.springerlink.com/ content/j213537rt4728519>. Acesso em: 8 set. 2009. doi: 10.1007/BF02602761.

LOPES, P.C.F. et al. Efeitos de diferentes frações inspiradas de oxigênio sobre a dinâmica respiratória em cães submetidos à infusão contínua de propofol e mantidos em ventilação espontânea. Brazilian Journal of Veterinary Resarch and Animal Science, São Paulo, v. 44, supl, p.30-37, 2007. Disponível em: <http://www.fumvet.com.br/novo/revista/44/ suplemento/30-37.pdf>. Acesso em: 8 set. 2009.

LOPES, P.C.F. Efeitos de diferentes frações inspiradas de oxigênio sobre $o$ índice biespectral, parâmetros respiratórios, hemogasométricos, hemodinâmicos e ecocardiográficos em cães submetidos à anestesia com infusão contínua de propofol e mantidos em ventilação espontânea. 2005. 169f. Dissertação (Mestrado em Cirurgia Veterinária) - Faculdade de Ciências Agrárias e Veterinárias, Universidade Estadual Paulista, Jaboticabal, SP.

LÓPEZ, A. Sistema respiratório. In: CARLTON, W.W.; MCGAVIN, M.D. Patologia veterinária especial de Thomson. 2.ed. Porto Alegre: Artmed, 1998. p.132-190.

LORING, S.H.; BUTLER, J.P. Gas exchange in body cavities. In: FARHI L.E.; TENNEY, S.M. Handbook of physiology. Section 3, the repiratory system. Bethesda, Maryland: American Physiology Society, 1987. p.283-295.

MAGNUSSON, L.; SPAHN, D.R. New concepts of atelectasis during generak anaesthesia. Bristish Journal of Anaesthesia, Oxford, v.91, n.1, p.61-72, 2003. Disponível em: <http:// bja.oxfordjournals.org/cgi/content/full/91/1/61>. Acesso em: 8 set. 2009. doi: 10.1093/bja/aeg085.

MILLER, R.D. Miller's anesthesia. 6.ed. Philadelphia: Elsevier Churchill Livingstone, 2005. 3024p.
NEUMANN, P. et al. Positive end-expiratory pressure prevents atelectasis during general anaesthesia even in the presence of a high inspired oxygen concentration. Acta Anaesthesiologica Scandinavica, Copenhagen, v.43, n.3, p.295-301, 1999. Disponível em: <http://www3.interscience.wiley.com/journal/ 119055152>. Acesso em: 8 set. 2009. doi: 10.1034/j.13996576.1999.430309.x.

NUNN J.F. Applied respiratory physiology. 2.ed. London: Bulterworths. 1979. 524p.

OTIS, D.R. et al. Role of pulmonary surfactant in airway closeure; a computational study. Journal of Applied Physiology, Bethesda, v.75, n.3, p.1323-1333, 1993. Disponível em: <http://jap.physiology.org/cgi/reprint/75/3/ 1323>. Acesso em: 8 set. 2009.

OYARZUN, M.J. et al. Factors affecting distribuition of alveolar surfactant during resting ventilation. American Journal of Physiology. Lung Cellular and Molecular Physiology, Bethesda, v.261, n.2, p.210-217, 1991. Disponível em: <http://ajplung.physiology.org/cgi/reprint/261/ 2/L210>. Acesso em: 8 set. 2009.

PASTEUR, W. Active lobar collapse of the lung after abdominal operations: a contribution to the study of post-operative lung complications. Lancet, London, v.176, n.4545, p.1080-1083, 1910.

PELOSI, P. et al. Respiratory system mechanics in sedated, paralyzed, morbidly obese patients. Journal of Applied Physiology, Bethesda, v.82, n.3, p.811-818, 1997. Disponível em: <http://jap.physiology.org/cgi/reprint/82/3/811>. Acesso em: 8 set. 2009 .

REBER, A. et al. Lung aeration and pulmonary gas exchange during lumbar epidural anaesthesia and in the lithotomy position in elderly patients. Anaesthesia, London, v.53, n.9, p.854861, 1998. Disponível em: <http://www3.interscience.wiley.com/ journal/119102138/abstract $>$. Acesso em: 8 set. 2009. doi: 10.1046/j.1365-2044.1998.00491.x.

REHDER, K. et al. Ventilation-perfusion relationship in young healthy awake and anesthetized-paralyzed man. Journal of Applied Physiology, Bethesda, v.47, n.4, p.745-753, 1979. Disponível em: <http://jap.physiology.org/cgi/reprint/47/4/ 745>. Acesso em: 8 set. 2009.

ROSA, A.L. et al. Right-to-left shunt determination in dog lungs under inhalation anesthesia with rebreathing and nonreabreathing system. Acta Cirurgica Brasileira, São Paulo, v.21, n.6, p.374-379, 2006. Disponível em: <http:// www.scielo.br/pdf/acb/v21n6/04.pdf>. Acesso em: 8 set. 2009. doi: 10.1590/S0102-86502006000600004.

ROTHEN, H.U. et al. Re-expansion of atelectasis during general anaesthesia: a computed tomography study. British Journal of Anaesthesia, Oxford, v.71, n.6, p.788-795 1993.

ROTHEN, H.U. et al. Influence of gas compositon on recurrence of atelctasis after reexpansion manuever during general anaeshtesia. Anesthesiology, Philadelphia, v.82, n.4, p.832842, 1995a. Disponível em: <http://journals.lww.com/ anesthesiology/Fulltext/1995/04000/Influence_of_Gas_ Composition_on_Recurrence_of.4.aspx $>$. Acesso em: 8 set. 2009. 
ROTHEN, H.U. et al. Prevention of atelctasis during general anaesthesia. Lancet, London, v.345, n.8962, p.1387-1391, $1995 b$.

ROTHEN, H.U. et al. Atelectasis and pulmonary shunting during induction of general anaesthesia - can they be avoided? Acta Anaesthesiologica Scandinavica, Copenhagen, v.40, n.5, p.524529, 1996. Disponível em: <http://www3.interscience.wiley.com/ journal/121450645/abstract>. Acesso em: 8 set. 2009. doi: 10.1111/j.1399-6576.1996.tb04483.x.

SENTÜRK, M. New concepts of management of one-lung ventilation. Current Opinion in Anaesthesiology, Philadelphia, v.19, n.1, p.1-4, 2006. Disponível em: <http://journals.lww.com/ c o-a nesthesiology/Abstract/2006/02000/ New_concepts_of_the_management_of_one_lung.2.aspx $>$. Acesso em: 8 set. 2009. doi: 10.1097/01.aco.0000192778.17151.2c.

STRANDBERG, A. et al. Atelectasis during anaesthesia and in the postoperative period. Acta Anaesthesiologica
Scandinavica, Copenhagen, v.30, n.2, p.154-158, 1986. Disponível em: <http://www3.interscience.wiley.com/journal/ 121490469/abstract>. Acesso em: 8 set. 2009. doi: 10.1111/ j.1399-6576.1986.tb02387.x.

TOKICS, L. et al. Computerized tomography of the chest and gas exchange measurements during ketamine anaesthesia. Acta Anaesthesiologica Scandinavica, Copenhagen, v.31, n.8, p.684692, 1987. Disponível em: <http://www3.interscience.wiley.com/ journal/121492067/abstract>. Acesso em: 8 set. 2009. doi: 10.1111/j.1399-6576.1987.tb02646.x.

WOLLMER, P. et al. Pulmonary clearance of 99mTc-DTPA during halotahane anesthesia. Acta Anaesthesiologica Scandinavica, Copenhagen, v.34, n.5, p.572-575, 1990. Disponível em: <http://www3.interscience.wiley.com/journal/ 121491644/abstract>. Acesso em: 8 set. 2009. doi: 10.1111/ j.1399-6576.1990.tb03147.x. 